\title{
Distributed Adaptive Controllers for Cooperative Output Regulation of Heterogeneous Agents over Directed Graphs
}

\author{
Zhongkui Li ${ }^{\text {a }}$, Michael Z. Q. Chen ${ }^{\mathrm{b}}$, Zhengtao Ding ${ }^{\mathrm{c}}$

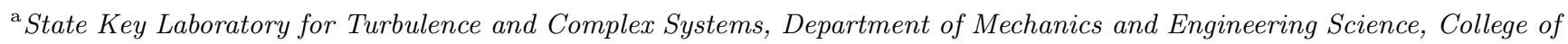 \\ Engineering, Peking University, Beijing 100871, China \\ ${ }^{\mathrm{b}}$ Department of Mechanical Engineering, The University of Hong Kong, Pokfulam Road, Hong Kong \\ ${ }^{\mathrm{c}}$ The Control Systems Centre, School of Electrical and Electronic Engineering, University of Manchester, Sackville Street \\ Building, Manchester M13 9PL, UK
}

\begin{abstract}
This paper considers the cooperative output regulation problem for linear multi-agent systems with a directed communication graph, heterogeneous linear subsystems, and an exosystem whose output is available to only a subset of subsystems. Both the cases with nominal and uncertain linear subsystems are studied. For the case with nominal linear subsystems, a distributed adaptive observer-based controller is designed, where the distributed adaptive observer is implemented for the subsystems to estimate the exogenous signal. For the case with uncertain linear subsystems, the proposed distributed observer and the internal model principle are combined to solve the robust cooperative output regulation problem. Compared with the existing works, one main contribution of this paper is that the proposed control schemes can be designed and implemented by each subsystem in a fully distributed fashion for a class of directed graphs.
\end{abstract}

Key words: Networked control systems, cooperative control, output regulation, consensus, directed graph.

\section{Introduction}

Cooperative output regulation of multi-agent systems is to have a group of autonomous agents (subsystems) interacting with each other via communication or sensing to asymptotically track a prescribed trajectory and/or maintain asymptotic rejection of disturbances. The cooperative output regulation problem is closely related to the consensus problem and other cooperative control problems as studied in $[11,5]$ and the references therein. A central work in cooperative output regulation is to design appropriate distributed controllers, using only the local state or output information of each agent and its neighbors. In recent years, many interesting results are reported on cooperative output regulation, e.g., in $[15,13,6,9,12,14,4,2]$. In particular, several state and output feedback control laws are proposed in $[15,13,6,9]$ to achieve cooperative output regulation for multi-agent

\footnotetext{
^ This work was supported by the National Natural Science Foundation of China under grants 61473005 and 11332001.

Email addresses: zhongkli@pku.edu.cn (Zhongkui Li), mzqchen@hku.hk (Michael Z. Q. Chen), zhengtao.ding@manchester.ac.uk (Zhengtao Ding).
}

systems with heterogeneous but known linear subsystems. The robust cooperative output regulation problem of uncertain linear multi-agent systems is studied in $[12,14]$, where internal-model-based controllers are designed. In [4,2], cooperative global output regulation is discussed for several classes of nonlinear multi-agent systems.

Although many advances have been reported on the cooperative output regulation problem, some challenging issues remain unresolved. For instance, control design presented in $[13,12,14]$ explicitly depends on certain nonzero eigenvalues of the Laplacian matrix associated with the communication graph. However, it is worth mentioning that any nonzero eigenvalue of the Laplacian matrix is global information of the communication graph. Using these global information of the communication graph prevents fully distributed implementation of the controllers. In other words, the controllers given in the aforementioned papers are not fully distributed. In [6], fully distributed adaptive controllers are proposed, which implement adaptive laws to update the time-varying coupling weights between neighboring agents. Similar adaptive protocols have been also presented in $[8,16,7]$ to solve the consensus problems. It is 
worth noting that the adaptive controllers in [6] are applicable to only the case where the graph among the agents are undirected and that the adaptive protocols in $[8,16,7]$ are designed for homogeneous multi-agent systems. To design fully distributed controllers to achieve cooperative output regulation for heterogeneous multiagent systems with general directed graphs is much more challenging, due to both the heterogeneity of the agents and the asymmetry of the directed graphs, and is still open, to the best knowledge of the authors.

This paper extends the fully distributed control design to the cooperative output regulation problem for linear multi-agent systems with a directed communication graph, heterogeneous linear subsystems, and an exosystem whose output is available to only a subset of subsystems. Both the cases with nominal and uncertain linear subsystems are studied. A distributed adaptive observer-based controller is designed to solve the cooperative output regulation problem for multi-agent systems with nominal linear subsystems. The distributed adaptive observer, which utilizes the observer states from neighboring subsystems, is constructed for the subsystems to asymptotically estimate the state of the exosystem. The case with uncertain linear subsystems is further studied. The proposed distributed adaptive observer and the internal model principle are combined to design distributed controllers to solve the robust cooperative output regulation problem. The proposed control schemes in this paper, in contrast to the controllers in $[13,12,14]$, can be designed and implemented by each subsystem in a fully distributed fashion, and, different from those in [6], are applicable to directed graphs.

\section{Cooperative Output Regulation Problem}

In this section, we consider a network consisting of $N$ heterogeneous subsystems and an exosystem. The dynamics of the $i$-th subsystem are described by

$$
\begin{aligned}
\dot{x}_{i} & =A_{i} x_{i}+B_{i} u_{i}+E_{i} v, \\
e_{i} & =C_{i} x_{i}+D_{i} v, \quad i=1, \cdots, N,
\end{aligned}
$$

where $x_{i} \in \mathbf{R}^{n_{i}}, u_{i} \in \mathbf{R}^{m_{i}}$, and $e_{i} \in \mathbf{R}^{p_{i}}$ are, respectively, the state, the control input, and the regulated output of the $i$-th subsystem, and $A_{i}, B_{i}, C_{i}$, and $D_{i}$ are constant matrices with appropriate dimensions. In (1), $v \in \mathbf{R}^{q}$ represents the exogenous signal which can be either a reference signal to be tracked or the disturbance to be rejected. The exogenous signal $v$ is generated by the following exosystem:

$$
\dot{v}=S v, y_{v}=F v,
$$

where $y_{v} \in \mathbf{R}^{l}$ is the output of the exosystem, $S \in \mathbf{R}^{q \times q}$, and $F \in \mathbf{R}^{l \times q}$.

To achieve cooperative output regulation, the subsystems need information from other subsystems or the exosystem. The information flow among the $N$ subsystems can be modeled by a directed graph $\mathcal{G}=(\mathcal{V}, \mathcal{E})$, where $\mathcal{V}=\left\{v_{1}, \cdots, v_{N}\right\}$ is the node set and $\mathcal{E} \subseteq \mathcal{V} \times \mathcal{V}$ is the edge set, in which an edge is represented by an ordered pair of distinct nodes. If $\left(v_{i}, v_{j}\right) \in \mathcal{E}$, node $v_{i}$ is called a neighbor of node $v_{j}$. A directed path from node $v_{i_{1}}$ to node $v_{i_{l}}$ is a sequence of adjacent edges of the form $\left(v_{i_{k}}, v_{i_{k+1}}\right), k=1, \cdots, l-1$. A directed graph contains a directed spanning tree if there exists a root node that has directed paths to all other nodes.

Since the exosystem (2) does not receive information from any subsystem, it can be viewed as a virtual leader, indexed by 0 . The $N$ subsystems in (1) are the followers, indexed by $1, \cdots, N$. Assume that the output $y_{v}$ of the exosystem (2) is available to only a subset of the followers. Without loss of generality, suppose that the subsystems indexed by $1, \cdots, M(1 \leq M \ll N)$, have direct access to the exosystem (2) and the rest of the followers do not. The followers indexed by $1, \cdots, M$, are called the informed followers and the rest are the uninformed ones. The communication graph $\mathcal{G}$ among the $N$ subsystems is assumed to satisfy the following assumption.

Assumption 1 For each uninformed follower, there exists at least one informed follower that has a directed path to that uninformed follower.

For the case with only one informed follower, Assumption 1 is equivalent to that the graph $\mathcal{G}$ contains a directed spanning tree with the informed follower as the root node. For the directed graph $\mathcal{G}$, its adjacency ma$\operatorname{trix} \mathcal{A}=\left[a_{i j}\right] \in \mathbf{R}^{N \times N}$ is defined by $a_{i i}=0, a_{i j}=1$ if $\left(v_{j}, v_{i}\right) \in \mathcal{E}$ and $a_{i j}=0$ otherwise. The Laplacian matrix $\mathcal{L}=\left[\mathcal{L}_{i j}\right] \in \mathbf{R}^{N \times N}$ associated with $\mathcal{G}$ is defined as $\mathcal{L}_{i i}=\sum_{j \neq i} a_{i j}$ and $\mathcal{L}_{i j}=-a_{i j}, i \neq j$.

Because the informed subsystems indexed by $1, \cdots, M$, can have direct access to the exosystem (2), they do not have to communicate with other subsystems to ensure that $e_{i}, 1, \cdots, M$, converge to zero. To avoid unnecessarily increasing the number of communication channels, we assume that the informed subsystems do not receive information from other subsystems, i.e., they have no neighbors except the exosystem. In this case, the Laplacian matrix $\mathcal{L}$ associated with $\mathcal{G}$ can be partitioned as $\mathcal{L}=\left[\begin{array}{cc}0_{M \times M} & 0_{M \times(N-M)} \\ \mathcal{L}_{2} & \mathcal{L}_{1}\end{array}\right]$ where $\mathcal{L}_{2} \in \mathbf{R}^{(N-M) \times M}$ and $\mathcal{L}_{1} \in \mathbf{R}^{(N-M) \times(N-M)}$. Under Assumption 1, it is known that all the eigenvalues of $\mathcal{L}_{1}$ have positive real parts [1]. Moreover, it is easy to verify that $\mathcal{L}_{1}$ is a nonsingular $M$-matrix [10].

The objective of the cooperative output regulation problem considered in this section is to design appropriate distributed controllers based on the local information available to the subsystems such that (i) The overall closed-loop system is asymptotically stable when $v=0$; (ii) For any initial conditions $x_{i}(0), i=1, \cdots, N$, and $v(0), \lim _{t \rightarrow \infty} e_{i}(t)=0$.

To solve the above cooperative output regulation problem, the following assumptions are needed, which are 
standard in the classic output regulation problem [3].

Assumption 2 The matrix $S$ has no eigenvalues with negative real parts.

Assumption 3 The pairs $\left(A_{i}, B_{i}\right), i=1, \cdots, N$, are stabilizable.

Assumption 4 The pair $(S, F)$ is detectable.

Assumption 5 For all $\lambda \in \sigma(S)$, where $\sigma(S)$ denotes the spectrum of $S, \operatorname{rank}\left(\left[\begin{array}{cc}A_{i}-\lambda I & B_{i} \\ C_{i} & 0\end{array}\right]\right)=n_{i}+p_{i}$.

Since the exogenous signal $v$ is not available to the subsystems for feedback control, the subsystems need to implement some observers to estimate $v$. For the informed subsystems that have direct access to the output $y_{v}$ of the exosystem (2), they can estimate $v$ by using the following observers:

$$
\dot{\xi}_{i}=S \xi_{i}+L\left(F \xi_{i}-y_{v}\right), \quad i=1, \cdots, M,
$$

where the feedback gain matrix $L \in \mathbf{R}^{p \times l}$ is chosen such that $S+L F$ is Hurwitz. Denote by $\bar{\xi}_{i}=\xi_{i}-v$ the estimation errors. From (2) and (3), it is easy to see that $\dot{\bar{\xi}}_{i}=(S+L F) \bar{\xi}_{i}, i=1, \cdots, M$, implying that $\lim _{t \rightarrow \infty} \vec{\xi}_{i}(t)=0, i=1, \cdots, M$.

For the uninformed subsystems that do not have direct access to (2), we need to construct distributed observers to estimate the exogenous signal $v$. The distributed adaptive observer for each uninformed subsystem is described by

$$
\begin{aligned}
& \dot{\xi}_{i}=S \xi_{i}-\left(d_{i}+\rho_{i}\right) \sum_{j=1}^{N} a_{i j}\left(\xi_{i}-\xi_{j}\right), \\
& d_{i}=\left[\sum_{j=1}^{N} a_{i j}\left(\xi_{i}-\xi_{j}\right)\right]^{T} \Gamma\left[\sum_{j=1}^{N} a_{i j}\left(\xi_{i}-\xi_{j}\right)\right],
\end{aligned}
$$

where $\xi_{i} \in \mathbf{R}^{p}, i=M+1, \cdots, N$, denotes the estimate of $v$ on the $i$-th uninformed subsystem, $d_{i}(t)$ denotes the time-varying coupling gain associated with the $i$-th uninformed subsystem with $d_{i}(0) \geq 0, a_{i j}$ is the $(i, j)$-th entry of the adjacency matrix associated with $\mathcal{G}, \Gamma \in \mathbf{R}^{l \times l}$ is the feedback gain matrix, and $\rho_{i}(\cdot)$ are smooth and monotonically increasing functions in terms of $\sum_{j=1}^{N} a_{i j}\left(\xi_{i}-\xi_{j}\right)$. The parameters $\Gamma$ and $\rho_{i}(\cdot)$ are to be determined.

Theorem 1 Suppose that Assumptions 1 and 4 hold. Then, $\lim _{t \rightarrow \infty}\left(\xi_{i}(t)-v(t)\right)=0, i=1, \cdots, N$, if $L$ in (3) is chosen such that $S+L F$ is Hurwitz and the parameters in the adaptive observer (4) is chosen to be $\Gamma=P^{2}$ and $\rho_{i}=\zeta_{i}^{T} P \zeta_{i}, i=M+1, \cdots, N$, where $\zeta_{i}=\sum_{j=1}^{N} a_{i j}\left(\xi_{i}-\xi_{j}\right)$ and $P>0$ is a solution to the algebraic Riccati equation (ARE):

$$
S^{T} P+P S+I-P^{2}=0 .
$$

Moreover, the coupling gains $d_{i}$ in (4) converge to some finite steady-state values.

Proof Let $\zeta=\left[\zeta_{M+1}^{T}, \cdots, \zeta_{N}^{T}\right]^{T}$. Then, $\zeta$ can be rewritten as

$$
\zeta=\left(\mathcal{L}_{2} \otimes I\right)\left[\begin{array}{c}
\bar{\xi}_{1} \\
\vdots \\
\bar{\xi}_{M}
\end{array}\right]+\left(\mathcal{L}_{1} \otimes I\right)\left[\begin{array}{c}
\bar{\xi}_{M+1} \\
\vdots \\
\bar{\xi}_{N}
\end{array}\right]
$$

where $\bar{\xi}_{i}=\xi_{i}-v$ denote the estimation errors. Because $\mathcal{L}_{1}$ is nonsingular and $\lim _{t \rightarrow \infty} \bar{\xi}_{i}(t)=0, i=1, \cdots, M$, it can be observed from (6) that $\lim _{t \rightarrow \infty} \bar{\xi}_{i}(t)=0, i=$ $M+1, \cdots, N$, if and only if $\lim _{t \rightarrow \infty} \zeta(t)=0$. From (4) and (6), it is not difficult to get that $\zeta$ and $d_{i}$ satisfy the following dynamics:

$$
\begin{aligned}
\dot{\zeta} & =\left[I \otimes S-\mathcal{L}_{1}(\widehat{D}+\hat{\rho}) \otimes I\right] \zeta+\left(\mathcal{L}_{2} \otimes L F\right) \bar{\xi}, \\
\dot{d}_{i} & =\zeta_{i}^{T} \Gamma \zeta_{i},
\end{aligned}
$$

where $\hat{\rho}=\operatorname{diag}\left(\rho_{M+1}, \cdots, \rho_{N}\right), \widehat{D}=\operatorname{diag}\left(d_{M+1}, \cdots, d_{N}\right)$, and $\bar{\xi}=\left[\bar{\xi}_{1}^{T}, \cdots, \bar{\xi}_{M}^{T}\right]^{T}$.

Let $V_{1}=\sum_{i=M+1}^{N}\left[\frac{q_{i}}{2}\left(2 d_{i}+\rho_{i}\right) \rho_{i}+\frac{\hat{\lambda}_{0}}{4} \tilde{d}_{i}^{2}\right]$, where $G \triangleq$ $\operatorname{diag}\left(q_{M+1}, \cdots, q_{N}\right)>0$ is chosen such that $G \mathcal{L}_{1}+$ $\mathcal{L}_{1}^{T} G>0[10,7], \hat{\lambda}_{0}$ denotes the smallest eigenvalue of $G \mathcal{L}_{1}+\mathcal{L}_{1}^{T} G$, and $\tilde{d}_{i} \triangleq d_{i}-\alpha$, with $\alpha$ being a positive constant. The time derivative of $V_{1}$ along the trajectory of (7) is given by

$$
\begin{aligned}
\dot{V}_{1}= & \sum_{i=M+1}^{N}\left[2 q_{i}\left(d_{i}+\rho_{i}\right) \dot{\rho}_{i}+q_{i} \rho_{i} \dot{d}_{i}+\frac{\hat{\lambda}_{0}}{2} \tilde{d}_{i} \zeta_{i}^{T} P^{2} \zeta_{i}\right] \\
\leq & \zeta^{T}\left[(\widehat{D}+\hat{\rho}) G \otimes\left(P S+S^{T} P\right)-\hat{\lambda}_{0}(\widehat{D}+\hat{\rho})^{2} \otimes P^{2}\right. \\
& \left.+\hat{\rho} G \otimes P^{2}+\frac{\hat{\lambda}_{0}}{2}(\widehat{D}-\alpha I) \otimes P^{2}\right] \zeta \\
& +\zeta^{T}\left[(\widehat{D}+\hat{\rho}) G \mathcal{L}_{2} \otimes P L F\right] \bar{\xi}
\end{aligned}
$$

where we have used the fact that $G \mathcal{L}_{1}+\mathcal{L}_{1}^{T} G \geq \hat{\lambda}_{0} I$. By using the well-known Young's inequality, we have

$$
\zeta^{T}\left(\hat{\rho} G \otimes P^{2}\right) \zeta \leq \zeta^{T}\left(\frac{\hat{\lambda}_{0}}{8} \hat{\rho}^{2} \otimes P^{2}+\frac{8}{\hat{\lambda}_{0}} G^{2}\right) \zeta
$$

and

$$
\begin{aligned}
\zeta^{T} & {\left[(\widehat{D}+\hat{\rho}) G \mathcal{L}_{2} \otimes P L F\right] \bar{\xi} } \\
& \leq \frac{\hat{\lambda}_{0}}{8}\|[(\widehat{D}+\hat{\rho}) \otimes P] \zeta\|^{2}+\frac{8}{\hat{\lambda}_{0}}\left\|\left(G \mathcal{L}_{2} \otimes L F\right) \bar{\xi}\right\|^{2} \\
& \leq \frac{\hat{\lambda}_{0}}{8}\|[(\widehat{D}+\hat{\rho}) \otimes P] \zeta\|^{2}+\frac{8}{\hat{\lambda}_{0}}\left\|G \mathcal{L}_{2} \otimes L F\right\|^{2}\|\bar{\xi}\|^{2} .
\end{aligned}
$$


Substituting (10) and (9) into (8) gives

$$
\begin{aligned}
\dot{V}_{1} \leq & \frac{1}{2} \zeta^{T}\left[(\widehat{D}+\hat{\rho}) G \otimes\left(P S+S^{T} P\right)\right] \zeta \\
& -\hat{\lambda}_{0} \zeta^{T}\left[\left(\frac{7}{8}(\widehat{D}+\hat{\rho})^{2}-\frac{1}{8} \hat{\rho}^{2}-\frac{1}{2} \widehat{D}+\frac{1}{2} \alpha I\right.\right. \\
& \left.\left.-\frac{8}{\hat{\lambda}_{0}^{2}} G^{2}\right) \otimes P^{2}\right] \zeta+\frac{8}{\hat{\lambda}_{0}}\left\|G \mathcal{L}_{2} \otimes L F\right\|^{2}\|\bar{\xi}\|^{2} \\
\leq & \frac{1}{2} \zeta^{T}\left[(\widehat{D}+\hat{\rho}) G \otimes\left(P S+S^{T} P\right)\right] \zeta \\
& -\hat{\lambda}_{0} \zeta^{T}\left[\left(\frac{1}{4}(\widehat{D}+\hat{\rho})^{2}+\hat{\alpha}\right) \otimes P^{2}\right] \zeta \\
& +\frac{8}{\hat{\lambda}_{0}}\left\|G \mathcal{L}_{2} \otimes L F\right\|^{2}\|\bar{\xi}\|^{2} \\
\leq & \frac{1}{2} \zeta^{T}\left[(\widehat{D}+\hat{\rho}) G \otimes\left(P S+S^{T} P-P^{2}\right)\right] \zeta \\
& +\frac{8}{\hat{\lambda}_{0}}\left\|G \mathcal{L}_{2} \otimes L F\right\|^{2}\|\bar{\xi}\|^{2}
\end{aligned}
$$

where we have chosen $\alpha \geq \max \frac{16 q_{i}^{2}}{\hat{\lambda}_{0}^{2}}+2 \hat{\alpha}$ and $\sqrt{\hat{\alpha}} \geq$ $\frac{1}{\hat{\lambda}_{0}} \max q_{i}$ to get the last two inequalities.

Let $V_{2}=\bar{\xi}^{T}\left(I_{M} \otimes \bar{Q}\right) \bar{\xi}$, where $\bar{Q}>0$ satisfy that $\bar{Q}(S+$ $L F)+(S+L F)^{T} \bar{Q}=-I$. The time derivative of $V_{3}$ along (??) can be obtained as

$$
\dot{V}_{2}=\bar{\xi}^{T}\left(I_{M} \otimes \bar{Q}\right) \dot{\bar{\xi}}=-\|\bar{\xi}\|^{2}
$$

Consider the Lyapunov function candidate $V=V_{1}+$ $h_{2} V_{2}$, where $h_{2} \geq \frac{6}{\hat{\lambda}_{0}}\left\|G \mathcal{L}_{2} \otimes L F\right\|^{2}$. Since $d_{i}(t) \geq 0$ for any $t>0$ and $\rho_{i}(\cdot)$ are monotonically increasing functions satisfying $\rho_{i}(s) \geq 0$ for $s>0$, it is not difficult to see that $V$ is positive definite with respect to $\bar{\xi}, \zeta_{i}$, and $\tilde{d}_{i}, i=M+1, \cdots, N$. By using (11) and (12), we can get the time derivative of $V$ as

$$
\dot{V}_{1} \leq-\frac{1}{2} \zeta^{T}[\widehat{D} \hat{\rho} G \otimes I] \zeta \leq-\frac{1}{2} \min q_{i}\|\zeta\|^{2} \leq 0
$$

From (13), we can get that each $d_{i}$ is bounded, which, by noting $\dot{d}_{i} \geq 0$, implies that each $d_{i}$ converges to some finite value. Note that $\dot{V}_{1} \equiv 0$ is equivalent to $\zeta \equiv 0$. By LaSalle's Invariance principle [10], it follows that $\zeta$ asymptotically converges to zero. That is, $\lim _{t \rightarrow \infty}\left(\xi_{i}(t)-v(t)\right)=0, i=M+1, \cdots, N$.

Remark 1 Theorem 1 shows that the local observer (3) and the distributed adaptive observer (4) ensure that the subsystems can asymptotically estimate the exogenous signal for general directed graphs satisfying Assumption 1. Because $(S, I)$ is controllable, the ARE (5) has a unique solution $P>0$. That is, the adaptive observer (4) always exists.

Upon the basis of the estimates $\xi_{i}$ of the exogenous signal $v$, we propose the following controller to each subsystem as

$$
u_{i}=K_{1 i} x_{i}+K_{2 i} \xi_{i}, \quad i=1, \cdots, N,
$$

where $K_{1 i} \in \mathbf{R}^{m_{i} \times n_{i}}$ and $K_{2 i} \in \mathbf{R}^{m_{i} \times q}$ are the feedback gain matrices. By substituting (14) into (1), we write the closed-loop dynamics of the subsystems as

$$
\begin{aligned}
\dot{x}_{i} & =\left(A_{i}+B_{i} K_{1 i}\right) x_{i}+E_{i} v+B_{i} K_{2 i} \xi_{i}, \\
e_{i} & =C_{i} x_{i}+D_{i} v, \quad i=1, \cdots, N
\end{aligned}
$$

Theorem 2 Suppose that Assumptions 1-5 hold. Select $K_{1 i}$ such that $A_{i}+B_{i} K_{1 i}$ are Hurwitz, and $K_{2 i}=U_{i}-$ $K_{1 i} X_{i}, i=1, \cdots, N$, where $\left(X_{i}, U_{i}\right)$ are unique solutions to the regulator equations ${ }^{1}$ :

$$
\begin{aligned}
X_{i} S & =A_{i} X_{i}+B_{i} U_{i}+E_{i}, \\
0 & =C_{i} X_{i}+D_{i}, \quad i=1, \cdots, N .
\end{aligned}
$$

Then, the cooperative output regulation problem is solved by the distributed controller (14) and the adaptive observers (3) and (4) constructed by Theorem 1.

Proof The closed-loop dynamics of each subsystem can be rewritten as

$$
\begin{aligned}
\dot{x}_{i} & =\left(A_{i}+B_{i} K_{1 i}\right) x_{i}+\left(E_{i}+B_{i} K_{2 i}\right) v+B_{i} K_{2 i} \bar{\xi}_{i}, \\
e_{i} & =C_{i} x_{i}+D_{i} v, \quad i=1, \cdots, N
\end{aligned}
$$

where $\bar{\xi}_{i}=\xi_{i}-v$ denote the estimation errors. Let $\tilde{x}_{i}=$ $x_{i}-X_{i} v, i=1, \cdots, N$. Then, by invoking (16), we can obtain from (17) and (2) that

$$
\begin{aligned}
\dot{\tilde{x}}_{i} & =\left(A_{i}+B_{i} K_{1 i}\right) \tilde{x}_{i}+B_{i} K_{2 i} \bar{\xi}_{i}, \\
e_{i} & =C_{i} \tilde{x}_{i}, \quad i=1, \cdots, N .
\end{aligned}
$$

Since $A_{i}+B_{i} K_{1 i}$ are Hurwitz and it follows from Theorem 1 that $\lim _{t \rightarrow \infty} \bar{\xi}_{i}(t)=0, i=1, \cdots, N$, it is easy to see that $\lim _{t \rightarrow \infty} \tilde{x}(t)=0$, which further implies that $\lim _{t \rightarrow \infty} e_{i}(t)=0, i=+1, \cdots, N$. That is, the cooperative output regulation problem is solved.

Remark 2 Theorem 2 states that the proposed adaptive control scheme consisting of the controller (14) and the observers (4) and (3) can solve the cooperative output regulation problem. Note that the proposed control scheme in this section, relying on the subsystem dynamics and the local information of neighboring subsystems, is fully distributed. By comparison, the controllers in the previous work [13] require some nonzero eigenvalue of the Laplacian matrix which is global information of the communication graph. The adaptive controllers in [6] are indeed fully distributed, which, however, are applicable to only undirected graphs. The proposed control scheme in this section works for directed graphs satisfying Assumption 1, whose design is more challenging.

${ }^{1}$ A necessary and sufficient condition for the solvability of (16) is Assumption 5 [3]. 


\section{Robust Cooperative Output Regulation Problem}

In this section, we consider the case where the subsystems in (1) are subject to uncertainties and have the same dimensions that are chosen to be $x_{i} \in \mathbf{R}^{n}$, $u_{i} \in \mathbf{R}^{m}$, and $e_{i} \in \mathbf{R}^{p}$. Specifically, the matrices in (1) can be written as

$$
\begin{aligned}
& A_{i}=\bar{A}_{i}+\Delta A_{i}, B_{i}=\bar{B}_{i}+\Delta B_{i}, E_{i}=\bar{E}_{i}+\Delta E_{i} \\
& C_{i}=\bar{C}_{i}+\Delta C_{i}, D_{i}=\bar{D}_{i}+\Delta D_{i}, i=1, \cdots, N,
\end{aligned}
$$

where $\bar{A}_{i}, \bar{B}_{i}, \bar{E}_{i}, \bar{C}_{i}, \bar{D}_{i}$ denote the nominal parts of these matrices, and $\Delta A_{i}, \Delta B_{i}, \Delta E_{i}, \Delta C_{i}, \Delta D_{i}$ are the uncertainties associated with these matrices. For convenience, let $\hat{\Delta}$ represents the uncertainty vector. Note that we assume that the model uncertainties are such that the closed-loop system remains stable.

For the uncertain subsystems described by (1) and (19) and the exosystem (2), the robust cooperative output regulation problem in this section is to design appropriate distributed controllers based on the local information such that there exists an open neighborhood $W$ of the origin, then for any $\hat{\Delta} \in W$ and any initial condition $x_{i}(0), i=1, \cdots, N$, and $v(0), \lim _{t \rightarrow \infty} e_{i}(t)=0$.

The internal model principle will be utilized to solve the robust cooperative output regulation problem. Using the estimates $\xi_{i}$ of the exogenous signal $v$ via the observers (3) and (4), we introduce the following distributed dynamic state feedback control law:

$$
\begin{aligned}
u_{i} & =K_{x i} x_{i}+K_{z i} z_{i} \\
\dot{z}_{i} & =G_{1} z_{i}+G_{2}\left(C_{i} x_{i}+D_{i} \xi_{i}\right), \quad i=1, \cdots, N
\end{aligned}
$$

where $z_{i} \in \mathbf{R}^{n_{z}}$ with $n_{z}$ to be specified later, the pair $\left(G_{1}, G_{2}\right)$ incorporates the $p$-copy internal model of the matrix $S$ (The definition of the $p$-copy internal model can be found in [3]), and $K_{x i}$ and $K_{z i}$ are the feedback gain matrices to be designed. By combining (20) and (1), we get the augumented closed-loop dynamics of the subsystems as

$$
\begin{aligned}
\dot{\eta}_{i} & =A_{c i} \eta_{i}+B_{c i} v+Y_{c i} \bar{\xi}_{i} \\
e_{i} & =C_{c i} \eta_{i}+D_{i} v, \quad i=1, \cdots, N
\end{aligned}
$$

where $\eta_{i}=\left[x_{i}^{T}, z_{i}^{T}\right]^{T}$, the estimation errors $\bar{\xi}_{i}$ are defined as (17), and $A_{c i}=\left[\begin{array}{cc}A_{i}+B_{i} K_{x i} & B_{i} K_{z i} \\ G_{2} C_{i} & G_{1}\end{array}\right], B_{c i}=\left[\begin{array}{c}E_{i} \\ G_{2} D_{i}\end{array}\right]$, $C_{c i}=\left[\begin{array}{ll}C_{i} & 0\end{array}\right]$, and $Y_{c i}=\left[\begin{array}{c}0 \\ G_{2} D_{i}\end{array}\right]$. The stabilizability of the augmented system (22) is guaranteed by Assmptions 3 and $5[3]$.

Theorem 3 Suppose that Assumptions 1-5 hold. Choose $K_{x i}$ and $K_{z i}$ such that $\left[\begin{array}{cc}\bar{A}_{i}+\bar{B}_{i} K_{x i} & \bar{B}_{i} K_{z i} \\ G_{2} \bar{C}_{i} & G_{1}\end{array}\right]$ are Hurwitz, $i=1, \cdots, N$ Then, the robust cooperative output regulation problem is solved by the distributed controller (20) and the adaptive observers (3) and (4) constructed by Theorem 1.

Proof Since the nominal forms of the system matrices $A_{c i}$ of (21), equal to $\left[\begin{array}{cc}\bar{A}_{i}+\bar{B}_{i} K_{x i} & \bar{B}_{i} K_{z i} \\ G_{2} \bar{C}_{i} & G_{1}\end{array}\right]$, are Hurwitz, there exists an open neighborhood $W$ such that for any $\hat{\Delta} \in W$, the state matrices $A_{c i}$ are also Hurwitz. Because $\left(G_{1}, G_{2}\right)$ incorporates a $p$-copy internal model of $S$, it follows from Lemma 1.27 of [3] that for any $\hat{\Delta} \in W$, there exist $X_{x i}$ and $X_{z i}$ such that

$$
\begin{aligned}
X_{x i} S & =\left(A_{i}+B_{i} K_{x i}\right) X_{x i}+B_{i} K_{z i} X_{z i}+E_{i}, \\
X_{z i} S & =G_{1} X_{z i}+G_{2}\left(C_{i} X_{x i}+D_{i}\right), \\
0 & =C_{i} X_{x i}+D_{i}, \quad i=1, \cdots, N .
\end{aligned}
$$

Let $X_{c i}=\left[\begin{array}{c}X_{x i} \\ X_{z i}\end{array}\right]$. Then, $(22)$ can be rewritten as

$$
\begin{aligned}
X_{c i} S & =A_{c i} X_{c i}+B_{c i}, \\
0 & =C_{c i} X_{c i}+D_{i}, \quad i=1, \cdots, N .
\end{aligned}
$$

Let $\tilde{\eta}_{i}=\eta_{i}-X_{c i} v, i=1, \cdots, N$. Then, we can obtain from (21), (23), and (2) that

$$
\begin{aligned}
\dot{\tilde{\eta}}_{i} & =A_{c i} \tilde{\eta}_{i}+Y_{c i} \bar{\xi}_{i}, \\
e_{i} & =C_{c i} \tilde{x}_{i}, \quad i=1, \cdots, N .
\end{aligned}
$$

From $(24)$, we see that $\lim _{t \rightarrow \infty} e_{i}(t)=0$ if $\lim _{t \rightarrow \infty} \tilde{\eta}_{i}(t)=$ 0 , the latter of which can be shown by following similar steps in the proof of Theorem 2 .

Remark 3 The robust cooperative output regulation problem is also studied in the previous works [14,12]. On contrary to those controllers in $[14,12]$, which depend on global information of the communication graph, one favorable feature of the proposed adaptive control scheme in this section is fully distributed. It is worth mentioning that related robust consensus tracking problems are considered in $[17,18]$ for integrator-type agents with disturbances and unmodelled dynamics. By contrast, general linear and uncertain linear agents are studied in this paper. The methods in $[17,18]$, differing from those in this paper, cannot be directly used to solve the cooperative output regulation problem here.

\section{Conclusion}

In this paper, we have presented several distributed adaptive observer-based controllers to solve the cooperative output regulation problem for multi-agent systems with nominal or certain linear subsystems and a linear exosystem. A distinct feature of the proposed adaptive controllers is that they can be designed and implemented by each subsystem in a fully distributed manner for directed graphs. This is the main contribution of this paper with respect to the existing related works. 


\section{References}

[1] Y. Cao, W. Ren, and M. Egerstedt. Distributed containment control with multiple stationary or dynamic leaders in fixed and switching directed networks. Automatica, 48(8):15861597, 2012.

[2] Z. Ding. Adaptive consensus output regulation of a class of nonlinear systems with unknown high-frequency gain. Automatica, 51:348-355, 2015.

[3] J. Huang. Nonlinear Output Regulation: Theory and Applications. SIAM, 2004.

[4] A. Isidori, L. Marconi, and G. Casadei. Robust output synchronization of a network of heterogeneous nonlinear agents via nonlinear regulation theory. IEEE Transactions on Automatic Control, 59(10):2680-2691, 2014.

[5] Z. Li and Z. Duan. Cooperative Control of Multi-Agent Systems: A Consensus Region Approach. CRC Press, Boca Raton, FL, 2014.

[6] S. Li, G. Feng, X. Guan, X. Luo, and J. Wang. Distributed adaptive pinning control for cooperative linear output regulation of multi-agent systems. In The 32nd Chinese Control Conference, pp. 6885-6890, 2013.

[7] Z. Li, G. Wen, Z. Duan, and W. Ren. Designing fully distributed consensus protocols for linear multi-agent systems with directed graphs. IEEE Transactions on Automatic Control, 60(4): 1152-1157, 2015.

[8] Z. Li, W. Ren, X. Liu, and L. Xie. Distributed consensus of linear multi-agent systems with adaptive dynamic protocols. Automatica, 49(7):1986-1995, 2013.

[9] Z. Meng, T. Yang, D. Dimarogonas, and K. Johansson. Coordinated output regulation of multiple heterogeneous linear systems. In The 52nd IEEE Conference on Decision and Control, pp. 2175-2180. 2013.

[10] Z. Qu. Cooperative Control of Dynamical Systems. Applications to Autonomous Vehicles. Springer-Verlag, London, UK, 2009.

[11] W. Ren, R. Beard, and E. Atkins. Information consensus in multivehicle cooperative control. IEEE Control Systems Magazine, 27(2):71-82, 2007.

[12] Y. Su, Y. Hong, and J. Huang. A general result on the robust cooperative output regulation for linear uncertain multiagent systems. IEEE Transactions on Automatic Control, 58(5):1275-1279, 2013

[13] Y. Su and J. Huang. Cooperative output regulation of linear multi-agent systems. IEEE Transactions on Automatic Control, 57(4):1062-1066, 2012.

[14] X. Wang, Y. Hong, J. Huang, and Z. Jiang. A distributed control approach to a robust output regulation problem for multi-agent linear systems. IEEE Transactions on Automatic Control, 55(12):2891-2895, 2010.

[15] J. Xiang, W. Wei, and Y. Li. Synchronized output regulation of linear networked systems. IEEE Transactions on Automatic Control, 54(6):1336-1341, 2009.

[16] W.W. Yu, W. Ren, W.X. Zheng, G.R. Chen, and J.H. Lü. Distributed control gains design for consensus in multi-agent systems with second-order nonlinear dynamics. Automatica, 49(7):2107-2115, 2013.

[17] G.Q. Hu. Robust consensus tracking for an Integratortype multi-agent system with disturbances and unmodelled dynamics. International Journal of Control, 84(1):1-8, 2011.

[18] G.Q. Hu. Robust consensus tracking of a class of secondorder multi-agent dynamic systems. Systems and Control Letters, 61(1):134-142, 2012. 